\title{
TUNEL-positive cells in the surgical border of an amputation due to infected diabetic foot
}

\author{
C. BEKKER-MÉNDEZ ${ }^{1}$, R.M. GUZMÁN-AGUILAR ${ }^{2}$, M.A. HERNÁNDEZ-CUETO ${ }^{3}$, S. HUERTA-YEPEZ ${ }^{1}$, \\ R.A. JARILLO-LUNA ${ }^{3}$, E. GONZÁLEZ-VEYRAND ${ }^{2}$ and C.R. GONZÁLEZ-BONILLA ${ }^{1}$ \\ ${ }^{1}$ Unidad de Investigación en Infectología e Inmunología; ${ }^{2}$ Servicio de Cirugía, \\ Hospital de Infectología 'Dr. Daniel Méndez Hernández', Centro Médico Nacional ‘La Raza', IMSS; \\ ${ }^{3}$ Laboratorio de Inmunología Celular y Molecular de la Escuela Superior de Medicina del IPN, DF, México
}

Received June 21, 2011; Accepted October 6, 2011

DOI: $10.3892 / \mathrm{mmr} .2011 .667$

\begin{abstract}
Diabetic infected foot is the outcome of progressive vascular and neurological damage caused by persistent chronic hyperglycemia. Due to acute hypoxia and infection, the tissues develop extensive necrosis and gangrene, which often require amputation. The decision regarding the level of amputation relies mainly on the personal experience of the surgeon who must identify the healthy tissue without necrosis. However, tissue cells under stress may succumb before clear evidence of necrosis is present. In this study, dying cells with DNA damage were identified in the necrotic lesions and surgical borders of amputations. Therefore, the main purpose of this study was to identify apoptosis in the surgical borders of amputations required to treat infected diabetic foot. Apoptosis was identified by terminal deoxynucleotidyl transferase-mediated bio-dUTP nick-end labeling (TUNEL) in the superficial and deep tissues of wounds, and in the surgical borders of 10 consecutive adult patients with diabetes mellitus type 2 (DM2) who underwent amputation due to infected diabetic foot. The severity of the disease was classified by the Acute Physiological and Chronic Health Evaluation II (APACHE II) score on admission, and laboratory data were collected and bacteriological cultures were obtained from the lesions. The ankle/arm blood pressure index was measured, the blood flow in the affected limb was evaluated by high-resolution ultrasonography and color Doppler and pulse oximetry were performed during surgery. A total of 5 males and 5 females, aged 45-84 years (58.8 \pm 14.1$)$, were included. The APACHE II score was 2-18 points $(8 \pm 5.7)$. A total of 9 patients developed sepsis and 2 succumbed. A total of 5 patients required above-ankle amputation, and 5 required toe disarticulation. The ankle/
\end{abstract}

Correspondence to: Dr César R. González Bonilla, Unidad de Investigación en Infectología e Inmunología, Hospital de Infectología 'Dr. Daniel Méndez Hernández', Centro Médico Nacional 'La Raza', IMSS, DF, México

E-mail: crgb@prodigy.net.mx

Key words: apoptosis, diabetic foot, vascular endothelium apoptosis arm blood pressure index ranged from 0.23-0.85 (0.51 0.23$)$. Apoptotic cells were found in ulcers and abscesses, and in areas without necrosis. In the surgical borders of the amputations, apoptotic cells were found in skeletal muscle, blood vessels and peripheral nerves, particularly Schwann cells. Morphometric analysis revealed that the extent of apoptosis was 2-3 logarithms higher in the surgical borders of the infected diabetic foot compared to the venous ulcers, which were used as the reference. In conclusion, apoptosis was identified in regenerating tissues within diabetic foot wounds and in the surgical borders of amputations, where the surgeon considered the tissues to be undamaged. This information suggests that apoptosis may be present before visible signs of necrosis appear in the diabetic foot and may be caused by hypoxia, acidosis or proinflammatory cytokines. The extent of apoptosis in tissues proximal to necrotic areas may anticipate the development of diabetic foot and help the surgeon to make decisions regarding the need and extent of amputation.

\section{Introduction}

Diabetes mellitus type 2 (DM2) is a major global public health problem. Approximately 170 million individuals have the disease worldwide and this figure is expected to increase to 366 million in the next 20 years (1). DM2 is a complex syndrome characterized by diverse alterations in the metabolism of lipids, carbohydrates and proteins, which result in dysfunction in insulin use in peripheral tissues. The disease is progressive and produces irreversible vascular and neurological impairment. Although the diabetic complications have numerous various causes, the major pathways involved in the development and progression of vascular and neurological damage include the process of advanced glycation caused by chronic hyperglycemia, metabolic alterations and increased oxidative stress $(2,3)$.

DM2 is a chronic and incapacitating disease, and is the main cause of polyneuropathy, chronic renal failure, and vision loss and blindness in adults $(4,5)$. Feet are particularly vulnerable to the chronic complications of diabetes. Chronic peripheral arterial occlusive disease of the lower limbs occurs 4 times more frequently in diabetic patients, and approximately two-thirds of cases of atherosclerotic gangrene 
in males are associated with diabetes $(6,7)$. The infected diabetic foot has multiple causes and is a syndrome defined by infection, ulceration and destruction of deep tissues of the foot associated with peripheral neurological and vascular damage (8).

Hyperglycemia is the main underlying cause of the neurological and endothelial vascular damage, but ischemia contributes to the persistence and spread of infection since it impedes tissue oxygenation and the flow of soluble factors and immune cells to the interstitial space (9). Trauma and wounds are the main risk factors for the development of diabetic infected foot (10), which may begin as small painless blisters of the skin caused by the continual rubbing of improperly fitting shoes (11). Patients with infected diabetic foot often require an amputation. Between 50 and $95 \%$ of nontraumatic amputations of the leg occur in diabetic patients. Approximately $40 \%$ of these patients will require a second amputation in the following 5 years, with a $50 \%$ mortality rate in the 3 years following the second amputation (12). Patients who undergo amputation have a higher probability of mortality owing to diverse diseases related to vascular damage (13).

The complexity of the infected diabetic foot causes difficulties for the clinician when deciding whether the limb could be preserved and the precise time and level of the amputation. The evaluation required to pursue a radical treatment often implies magnitude of initial surgical procedure and is usually based on clinical criteria, including the spread and systemic implications of the infection and the vascular condition of the limb. The final decision typically depends on the experience of the surgeon $(6,9,12)$.

The tissues in a diabetic infected foot may exhibit apoptosis before changes caused by necrosis are evident. In the present study, we evaluated the skin, muscle, blood vessels and nerves in the necrotic lesions and surgical borders of amputations. The extension of apoptosis into tissues considered free of infection could be an early indicator of tissue damage, might anticipate the evolution of an amputation stump and could assist the surgeon in deciding on the need for and extent of amputation.

\section{Materials and methods}

Patients. Patients hospitalized in the General Surgery Service at the Infectious Diseases Hospital (Hospital de Infectología, Centro Médico 'La Raza', Instituto Mexicano del Seguro Social) were studied. Ten consecutive patients with DM2 and diabetic foot syndrome, but without multiple organ dysfunction, were included.

A clinical history was obtained, and a complete physical examination and presurgery laboratory tests were performed for each patient upon admission. The severity of the disease was evaluated using the Acute Physiology and Chronic Health Evaluation II (APACHE II) scale. The extension of infection and necrosis in the affected limb was determined using the Wagner classification of diabetic foot ulcers (14). Other laboratory tests included blood cell count, liver function test, gasometry, coagulation test and measurement of creatine phosphokinase and serum electrolyte levels. A total of 8 patients required amputation in the $48 \mathrm{~h}$ following admission. Information on the amputation level, microorganisms isolated from cultures, length of hospital stay and presence of complications were also recorded.

\section{Evaluation of blood flow in the affected limb}

Doppler ultrasound. The vascular state of the affected limb was evaluated by high-resolution vascular ultrasonography (color Doppler imaging) using a linear array of transducer elements of 7.5 MHz (Sonolayer Toshiba, model SSA-270A). The study was performed with the patient in the dorsal position, and the flow patterns were appraised in the peripheral arteries near their bifurcations and proximal ends. Information was obtained on the arterial intima and media thickness, presence and characteristics of atheromatous plaques and blood flow speed.

Ankle-brachial pressure index. The reduction in arterial blood flow in the legs was estimated by the ankle-brachial pressure index, which was calculated by dividing the systolic arterial blood pressure in each ankle (the highest value between the pedal and tibial arteries was selected) by the highest systolic value in the two brachial arteries. The lower of two different measurements was recorded.

Pulse oximetry. Pulse oximetry was performed throughout the surgery using a pulse oximeter (Datex Ohmeda Ltd., Stirling, UK) placed on the first or second toe.

Tissue collection and processing. Biopsies were obtained from the lesions and surgical borders during the amputation by excising a triangular sample of approximately $0.5 \mathrm{~cm}^{3}$ that included skin, subcutaneous soft tissue and muscle. The samples were fixed with $10 \%$ paraformaldehyde in $0.1 \mathrm{M}$ phosphate-buffered saline (PBS) $(\mathrm{pH} 7.2)$ and embedded in paraffin. Tissues were sectioned $(5 \mu \mathrm{m})$ and placed on special extra adhesive slides (Menzel-Glaser $\mathrm{GmbH}$ ). The slides were stained with hematoxylin and eosin (H\&E) and observed under light microscopy (Olympus BX40).

Measurement of apoptosis. Cells in late-stage apoptosis were identified by terminal deoxynucleotidyl transferase-mediated bio-dUTP nick-end labeling (TUNEL) using a commercial kit (In Situ Cell Death Detection kit, POD, Roche Applied Science, Mannheim, Germany) according to the manufacturer's instructions. As positive and negative internal controls, samples of colon cancer and normal skin were used (data not shown). To assess the extent of apoptosis, 5 patients with varicose ulcers were included as a reference. Briefly, the slides containing tissue samples were incubated with the enzyme terminal deoxynucleotidyl transferase at $37^{\circ} \mathrm{C}$ for $1 \mathrm{~h}$ and washed 3 times with $0.01 \mathrm{M}$ PBS. The anti-fluorescein-POD conjugate was added, and the slides were incubated for $30 \mathrm{~min}$ at $37^{\circ} \mathrm{C}$. Finally, the slides were developed with diaminobenzidine (DAB). The brown color of positive cells was observed with light microscopy. The intensity of staining of the TUNEL-positive cells was quantified in 4 different fields selected randomly on each slide using an image analyzer, the cutoff point for the positive staining was set using the software Image-Pro Plus ${ }^{\circledR}$ (Media Cybernetics, Inc., Silver Spring, MD, USA).

Statistical analysis. Arithmetic means and standard deviations of the clinical, laboratory and histological data were obtained 
and analyzed using the software GraphPad Prism ${ }^{\circledR}$ ver. 4.0 (GraphPad Software, Inc.).

\section{Results}

Description of the patients. With the aim to identify apoptosis of various cell types in tissue lesions and surgical borders of amputations, a descriptive study was performed on 10 consecutive patients who required amputation at different levels due to infected diabetic foot and who were admitted to the General Surgery Service. The general information of the patients is shown in Table I. Five males and females, respectively, were included. Their ages ranged from 45 to 85 years $(58.8 \pm 14.1)$. A total of 5 patients had a history of smoking and 3 of alcohol consumption; 1 patient was obese and 3 had a history of hypertension. A total of 7 patients had intermittent claudication and 4 were referred due to trauma to the foot. A total of 2 patients denied they were diabetic at the time of admission to the hospital, and the remainder had a 2- to 24-year history of DM2 (10.1 \pm 7.8 years). The foot infections had commenced 2-60 days prior to hospitalization (21.1 \pm 17.5 days).

The initial physical examination revealed the following crucial findings: a total of 9 patients exhibited swelling and violaceous skin, 5 presented with blisters with serous-sanguineous fluid, 8 displayed necrotic areas discharging pus, 5 had ulcers and 4 presented with subcutaneous crepitation. The

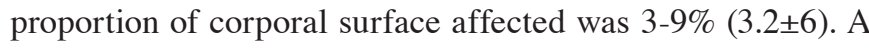
total of 5 patients presented with extensive gangrene comprising the whole foot, and the remainder had localized gangrene. The APACHE II score was 2-18 points $(8 \pm 5.7)$ at admission.

Findings of the laboratory tests at admission. Laboratory tests of infected diabetic foot patients are shown in Table II. The complete blood cell count revealed anemia in 7 patients, 1 of whom had severe anemia (hemoglobin concentration $=10.4 \pm 3.4 \mathrm{~g} / \mathrm{dl}$ ). A total of 8 patients had leukocytosis $\left(17.2 \pm 8.2 \times 10^{3}\right.$ cells $\left./ \mathrm{mm}^{3}\right)$, and 1 exhibited thrombocytopenia $\left(209.5 \pm 141.3 \times 10^{3}\right.$ cells/ $\mathrm{mm}^{3}$ ). Other laboratory tests revealed coagulation alterations (prothrombin, 69.3 $\pm 22.3 \%$; fibrinogen, $450.4 \pm 228.0 \mathrm{mg} / \mathrm{dl}$ ). A total of 7 patients presented with altered blood electrolyte levels: 3 with hyponatremia and 2 with hypernatremia (serum sodium concentration, $138.1 \pm 8.6 \mathrm{mEq} / \mathrm{l}), 5$ patients had hypokalemia (serum potassium, 3.6 $\pm 1.0 \mathrm{mEq} / \mathrm{l}$ ) and all had high calcium levels (serum calcium, $7.3 \pm 0.6 \mathrm{mg} / \mathrm{dl}$ ). All patients presented with serum albumin and total protein levels below the normal values $(2.2 \pm 0.4$ and $4.7 \pm 0.8 \mathrm{~g} / \mathrm{dl}$, respectively). In total, 5 patients had serum creatinine concentration above normal values $(3.3 \pm 3.0 \mathrm{mg} / \mathrm{dl})$, and 5 exhibited high urea concentration $(60.0 \pm 38.6 \mathrm{mg} / \mathrm{dl})$. A total of 6 patients had hyperglycemia $(162.4 \pm 82.7 \mathrm{mg} / \mathrm{dl})$. Serum cholesterol concentration and liver function tests were normal at the time of admission (data not shown).

Biopsies of muscle and aponeurosis were obtained from the affected and normally appearing tissues surrounding the lesions. All cultures from these samples revealed bacterial infection, and Candida albicans was isolated from 1 patient. Gram-negative bacterial infections prevailed. In 6 patients a single microorganism was isolated, and 2 microorganisms were isolated in 4 patients. A total of 14 different microorganisms were isolated: Staphylococcusaureus (3 patients),
Klebsiella pneumoniae (3 patients), Streptococcus pyogenes (2 patients), Escherichia coli (2 patients), Pseudomonas aeruginosa (2 patients), Enterobacter cloacae (1 patient) and Candida albicans (1 patient) (Table I).

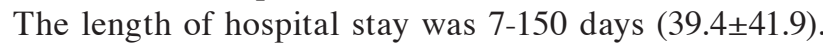
The medical treatment required the use of a broad spectrum of antibiotics, mainly third-generation cephalosporins. A total of 2 patients received a single antibiotic, 7 were treated with 2 antibiotics and 1 patient with 4 antibiotics. A total of 5 patients required above-ankle amputation and 5 toe disarticulation. All patients required several debridement procedures. Almost all patients developed complications: 9 patients developed sepsis, 7 acute renal failure and 1 liver insufficiency. A total of 8 patients improved and 2 succumbed, giving a mortality rate of $20 \%$ (Table I).

A total of 8 patients were admitted with normal blood pressure values, 1 patient presented with hypotension and 1 hypertension (systolic pressure 102.0 \pm 18.7 , diastolic pressure $64.5 \pm 13.8 \mathrm{mmHg}$ ). A total of 9 patients revealed abnormal blood pH, 2 of them with high and 7 with low values $(7.33 \pm 0.15)$. Only 1 patient had normal oxygen pressure and the remainder had low values $(52.5 \pm 20.7 \mathrm{mmHg})$. The ankle-brachial pressure index fluctuated between 0.23 and $0.85(0.51 \pm 0.23)$ (Table III).

The color Doppler ultrasonography revealed severe occlusion in 5 patients. The femoral and popliteal arteries were affected in 3 patients, respectively. The tibial and pedal arteries were affected in 2 patients. A total of 7 patients displayed atheromatous plaque causing $>50 \%$ occlusion and 3 patients had plaque without stenosis. In 7 patients, the pelvic extremities had diminished caliber of the posterior tibial and dorsalis pedis arteries, 6 patients had partial obstruction of the popliteal artery and 1 patient had total obstruction of the blood flow (Table III). The determining lesion of the acute event was located in the arteries: $31 \%$ in the femoral artery, $31 \%$ in the popliteal artery, $23 \%$ in the tibial artery and $15 \%$ in the tibial artery and dorsalis pedis artery. Oxygen saturation in the affected limb determined by pulse oximetry during the surgery was $20-75 \%(52.5 \pm 20.7)$; only 3 patients had $75 \% \mathrm{O}_{2}$ saturation (Table III).

Histopathological observations. The histopathological alterations and the presence of apoptosis in diverse tissues were examined in the lesions and surgical borders of the amputation. Fig. 1 shows representative images of the findings in the lesions, which were mainly ulcers and abscesses with inflammatory infiltration by polymorphonuclear leukocytes and granulation tissue with growth of new blood vessels (Fig. 1A-D). The epidermal borders displayed irregular hyperplasia with disordered cellular migration to the necrotic and fibrotic tissues. Keratinocytes exhibited abnormal nucleus:cytoplasm ratio and nuclear atypia. The dermis exhibited broad destruction caused by the inflammatory necrotic process. The epidermis exhibited atrophy and hyperkeratosis, and the dermis displayed elastotic degeneration. The connective tissue and skeletal muscle exhibited extensive areas of necrosis with inflammatory infiltration and irregular collagen deposition (Fig. 1E-H). Arteriolar vessels exhibited hyaline hyperplasia of the muscular layer. The small arteries displayed hyperplasia of the muscular layer, fibrosis, Monckeberg medial 


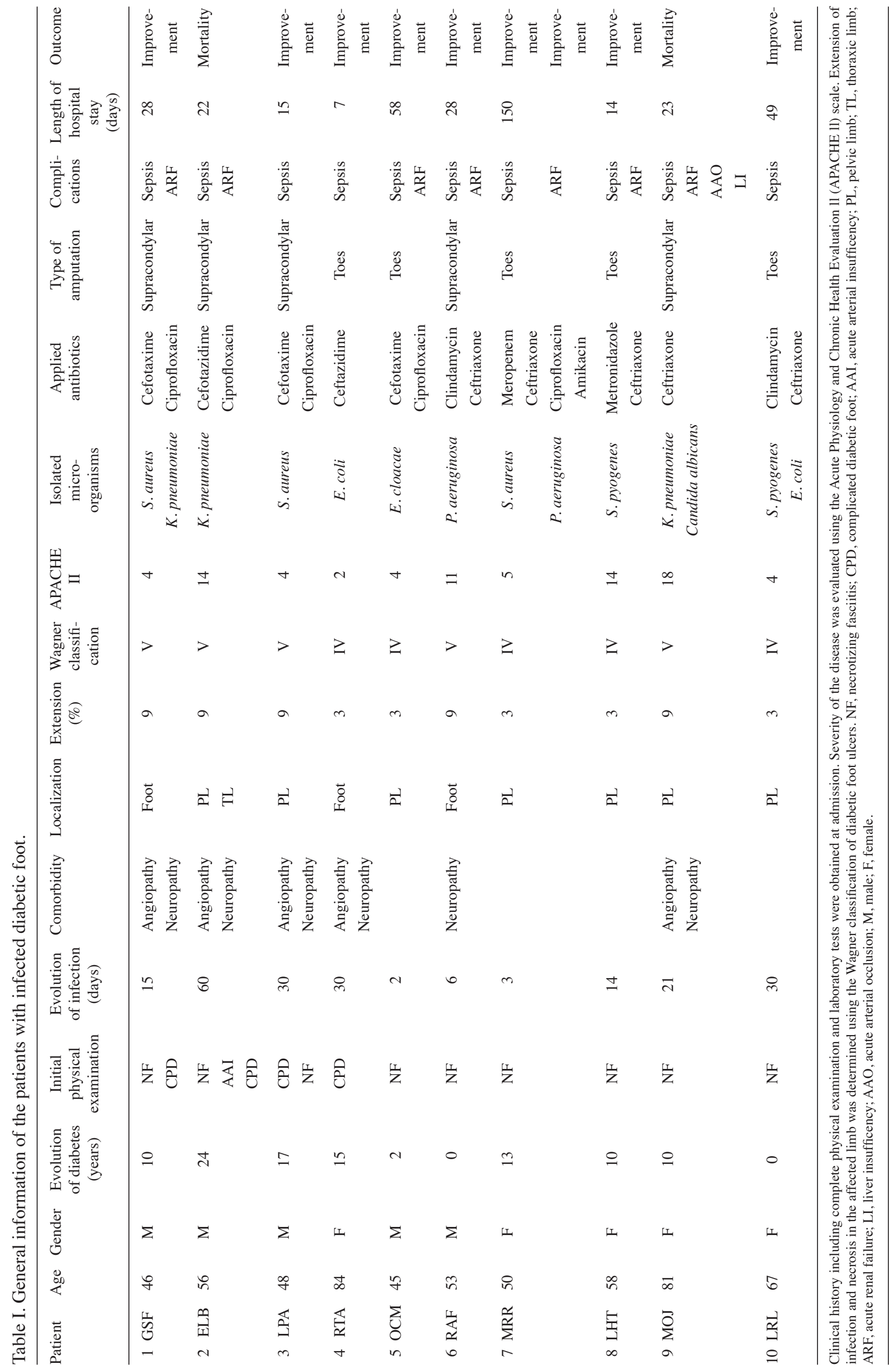




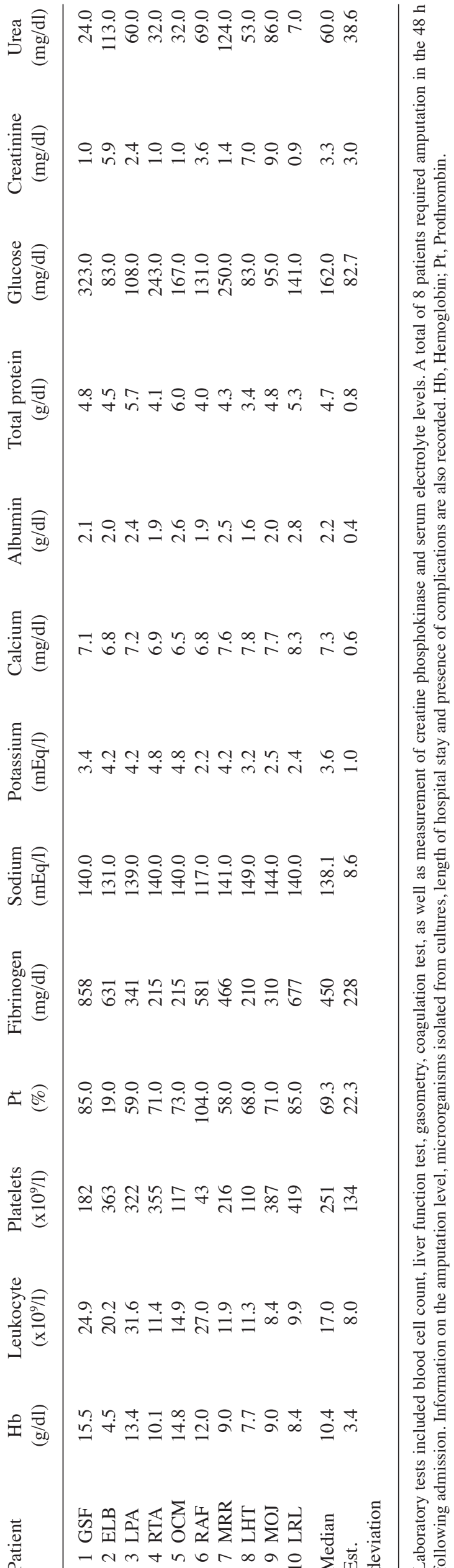

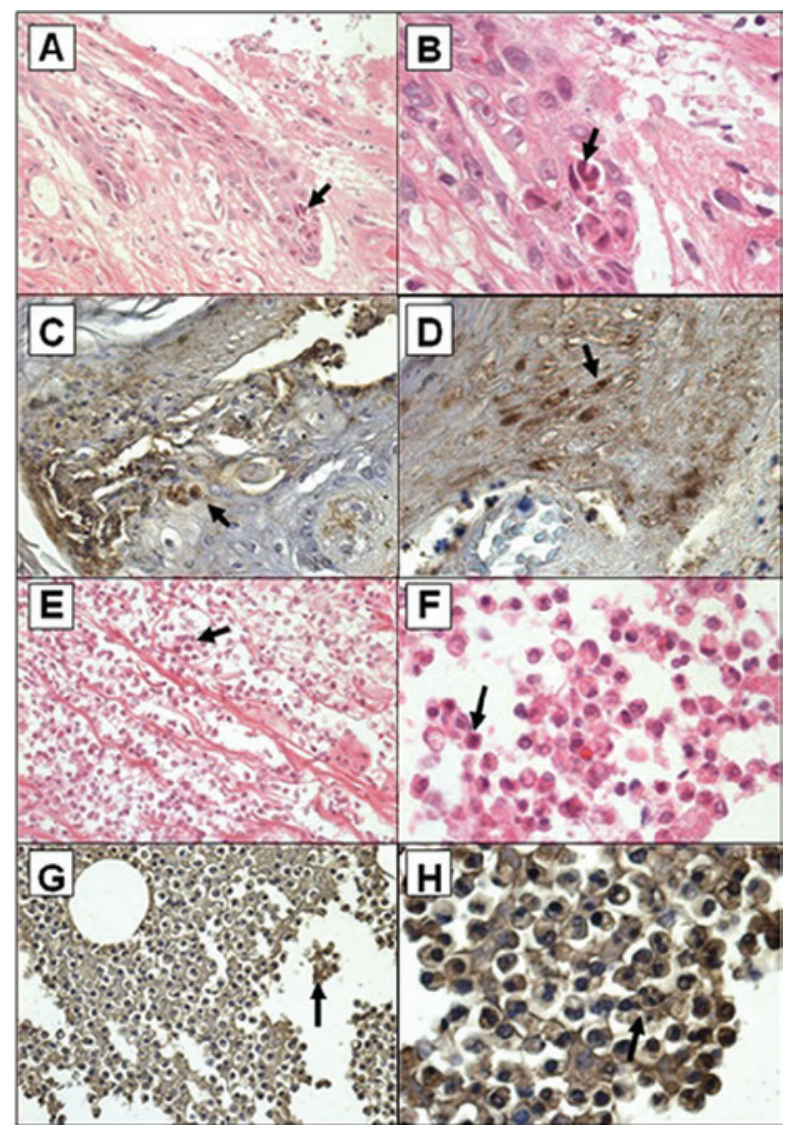

Figure 1. Representative images of lesions of infected diabetic foot tissues. H\&E staining and TUNEL immunohistochemical staining. (A and B) H\&E and (C and D) TUNEL-stained epidermal borders revealed irregular hyperplasia with disordered cellular migration to the necrotic and fibrotic tissues. Keratinocytes exhibited nuclear atypia and typical apoptotic changes, apoptotic bodies show strong expression for TUNEL (arrows). (E and F) H\&E and ( $\mathrm{G}$ and $\mathrm{H}$ ) TUNEL-stained abscesses with inflammatory infiltrate of polymorphonuclear leukocytes also with strong TUNEL-expression (arrows) Nuclear staining is representative of DNA fragmentation. (A,C,E and G) Magnification, $\mathrm{x} 40$ with (B,D,F and H) x100.

calcific sclerosis and atherosclerotic plaques with a noticeable decrease in the size of the vascular lumen. The venules also exhibited fibrotic changes, and the peripheral nerves exhibited broad atrophy of axons and Schwann cells. Apoptotic cells were found in the ulcers and abscesses (Fig. 1C and D; G and $\mathrm{H}$, respectively). TUNEL-positive cells, with characteristic nuclear changes, were observed in regenerating tissues; these cells included keratinocytes, fibroblasts, polymorphonuclear cells, foamy macrophages and endothelial cells. In areas without necrosis, some TUNEL-positive nuclear detritus was also observed.

Representative images of the histological findings at the surgical borders of the amputations. The skin displayed minor morphological changes, including slight hyperplasia, acanthosis and focal hyperpigmentation of the cells at the basal epidermal layer (Fig. 2). Certain keratinocytes revealed nuclear changes including condensation, size reduction, irregular outlines and fragmentation (Fig. 2A and B). Adipose and connective tissues did not exhibit significant alterations, but the skeletal muscle tissue displayed atrophy with similar nuclear 
Table III. Vascular evaluation of patients with infected diabetic foot.

\begin{tabular}{|c|c|c|c|c|c|}
\hline Patient & $\mathrm{pH}$ & $\begin{array}{l}\text { Ankle systolic arterial } \\
\text { pressure }(\mathrm{mmHg})\end{array}$ & $\mathrm{AB}$ index & $\begin{array}{l}\text { Color-doppler } \\
\text { ultrasonographic findings }\end{array}$ & $\begin{array}{c}\mathrm{O}_{2} \text { saturation } \\
(\%)\end{array}$ \\
\hline $1 \mathrm{GSF}$ & 7.39 & 60 & 0.40 & PAO popliteal, TAO tibial-fibular trunk & 20.0 \\
\hline 2 ELB & 7.56 & 45 & 0.42 & PAO popliteal, TAO tibial fibular trunk & 20.0 \\
\hline 3 LPA & 7.25 & 60 & 0.44 & PAO popliteal, TAO tibial-fibular trunk & 40.0 \\
\hline 4 RTA & 7.33 & 70 & 0.64 & PAO tibial-fibular trunk & 55.0 \\
\hline $5 \mathrm{OCM}$ & 7.38 & 85 & 0.77 & PAO pedia & 75.0 \\
\hline 6 RAF & 7.31 & 30 & 0.23 & PAO popliteal, TAO tibial-fibular trunk and pedia & 55.0 \\
\hline 7 MRR & 7.51 & 110 & 0.85 & Atheroma plaques without stenosis & 75.0 \\
\hline 8 LHT & 7.31 & 45 & 0.40 & PAO popliteal, TAO tibial-fibular trunk & 60.0 \\
\hline $9 \mathrm{MOJ}$ & 7.29 & 30 & 0.23 & TAO popliteal, tibial-fibular trunk and pedia & 50.0 \\
\hline $10 \mathrm{LRL}$ & 7.34 & 90 & 0.86 & Atheroma plaques without stenosis & 75.0 \\
\hline Median & 7.37 & 63 & 0.52 & & 52.5 \\
\hline $\begin{array}{l}\text { Est. } \\
\text { deviation }\end{array}$ & 0.10 & 26 & 0.24 & & 20.7 \\
\hline
\end{tabular}

Vascular state of the affected limb was evaluated by high-resolution vascular ultrasonography (color Doppler imaging). Arterial blood flow in the legs was estimated by the $\mathrm{AB}$ index, which was calculated by dividing the systolic arterial blood pressure in each ankle (the highest value between the pedal and tibial arteries was selected) by the highest systolic value in both brachial arteries. PAO, partial arterial obstruction; TAO, total arterial obstruction; AB index, ankle-brachial pressure index.

changes to those observed in keratinocytes. TUNEL-positive cells were observed in the dermis (Fig. 2A and B) and skeletal muscle (Fig. 2E-H). The blood vessels exhibited hyperplasia of the muscular layer with fibrosis, calcified plaques and partial occlusion. The endothelial cells also displayed nuclear changes, including condensation and fragmentation, and some were TUNEL positive (Fig. 2I-L). The peripheral nerves also exhibited fibrosis in all layers, and certain cells, including Schwann, exhibited nuclear condensation with irregular outlines and appeared to be TUNEL positive (Fig. 2M-P).

To provide a reference for quantifying the extent of the apoptotic process in the surgical borders of the amputations, the tissues in the diabetic patients were compared with varicose ulcers in non-diabetic patients. Fig. 3 shows images of the alterations in varicose ulcers. The epidermis adjacent to the ulcer borders exhibited keratinocyte hyperplasia (Fig. 3A and B) with focal morphological changes compatible with apoptosis (arrow). Although the dark staining due to melanin may be misjudged as apoptosis, certain cells were clearly TUNEL positive (Fig. 3C and D). Transverse sections of skeletal muscle revealed adipose infiltration and muscular atrophy (Fig. 3E and F), but TUNEL-positive cells were not identified (Fig. $3 \mathrm{G}$ and $\mathrm{H}$ ). The blood vessels (arterioles) had increased wall thickness due to hyperplasia of the muscular layer, fibrosis, and stromal reactive changes (Fig. 3I and J); however, they did not show TUNEL-positive cells (Fig. 3K and L). By contrast, venules demonstrated marked varicose changes such as endothelial ulceration and loss of the muscular layer. Peripheral nerves exhibited fibrosis, with fewer axons and Schwann cells (Fig. 3M and N). Similar to the other tissues, TUNEL-positive cells were not observed.

Computerized morphometric analysis of the TUNEL-positive cells in various tissues of the surgical borders of the amputa- tion required to treat diabetic foot compared with the samples from varicose ulcers. The magnitude of apoptosis was more variable in the skin and nerves of the amputees, although this difference was not significant when compared between all tissues (skin, muscle, blood vessels and nerves) (Fig. 4). However, the magnitude of apoptosis was 2-3 logarithms higher at the surgical borders of the amputations compared to the edges of varicose ulcers; this difference was more noticeable in nerves, blood vessels and muscle.

\section{Discussion}

Diabetic infected foot is one of the most severe, debilitating and life-threatening acute complications of DM2. Diabetic infected foot is caused by progressive vascular and neurological damage, immunodeficiency, impaired wound healing, orthopedic alterations and infection.

The diabetic infected foot is characterized by necrosis and gangrene resulting from ischemia and infection. The infection may spread quickly despite intensive medical care and often requires multiple surgical debridement and amputation. If untreated, the diabetic infected foot is capable of causing necrotizing fasciitis, a systemic inflammatory response and multiorgan failure. The decision about the amputation level is complex since the surgeon must balance the need to remove all necrotic tissue while maintaining the functionality of the stump as much as possible. Although higher level amputations are more likely to heal, it is often preferable to amputate as distally as possible to provide a functional stump. The long-term viability of a stump depends largely on the woundhealing ability and progression of vascular impairment.

Apoptosis may be an indicator of early tissue damage before the wound-healing process begins. We chose to investigate the presence of apoptosis in the tissues that a surgeon 


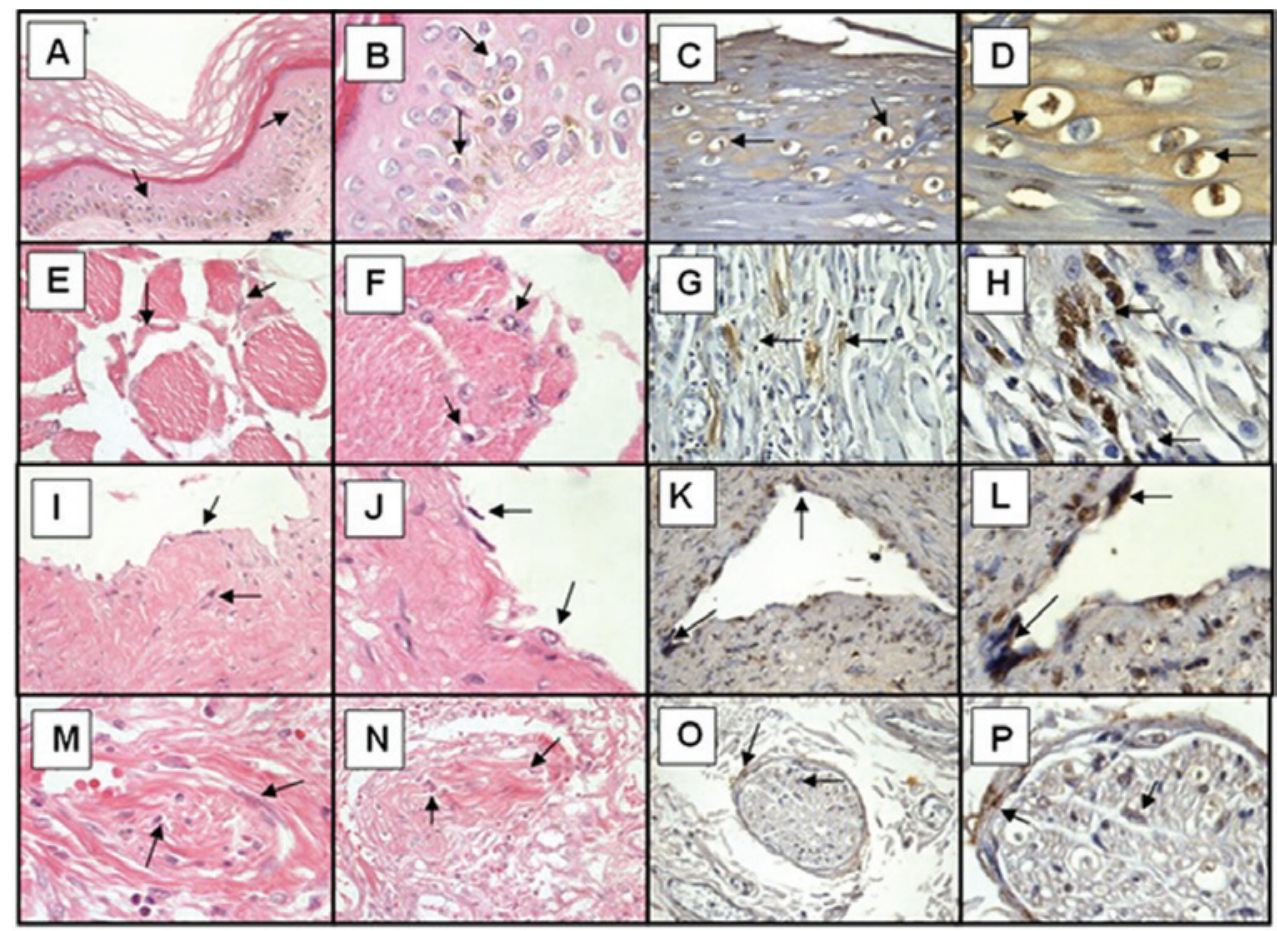

Figure 2. Representative images of the histological findings at the surgical borders of infected diabetic foot tissues. H\&E staining and TUNEL immunohistochemical staining. (A and B) H\&E and (C and D) TUNEL-stained epidermal borders exhibited minor morphological changes. Keratinocytes displayed apoptotic changes with mild TUNEL-expression in the nucleus and cytoplasm (arrows). (E and F) H\&E and (G and H) TUNEL-stained skeletal muscle with displayed atrophy with apoptotic changes; TUNEL-positive (arrows). (I and J) H\&E and (K and L) TUNEL-stained blood vessels displayed hyperplasia of the muscular layer with fibrosis and partial occlusion. The endothelial cells also revealed nuclear changes, including condensation and fragmentation, some of which were markedly TUNEL positive. ( $\mathrm{M}$ and $\mathrm{N}$ ) H\&E and ( $\mathrm{O}$ and $\mathrm{P}$ ) TUNEL-stained peripheral nerves with diabetic neuropathy also exhibited fibrosis in all layers and some cells, including Schwann, displayed nuclear condensation with irregular nuclear membrane, TUNEL-positive. (Magnification x40: A, C, E, G, I, K, M and O). (Magnification, x100: B, D, F, H, J, L and P).

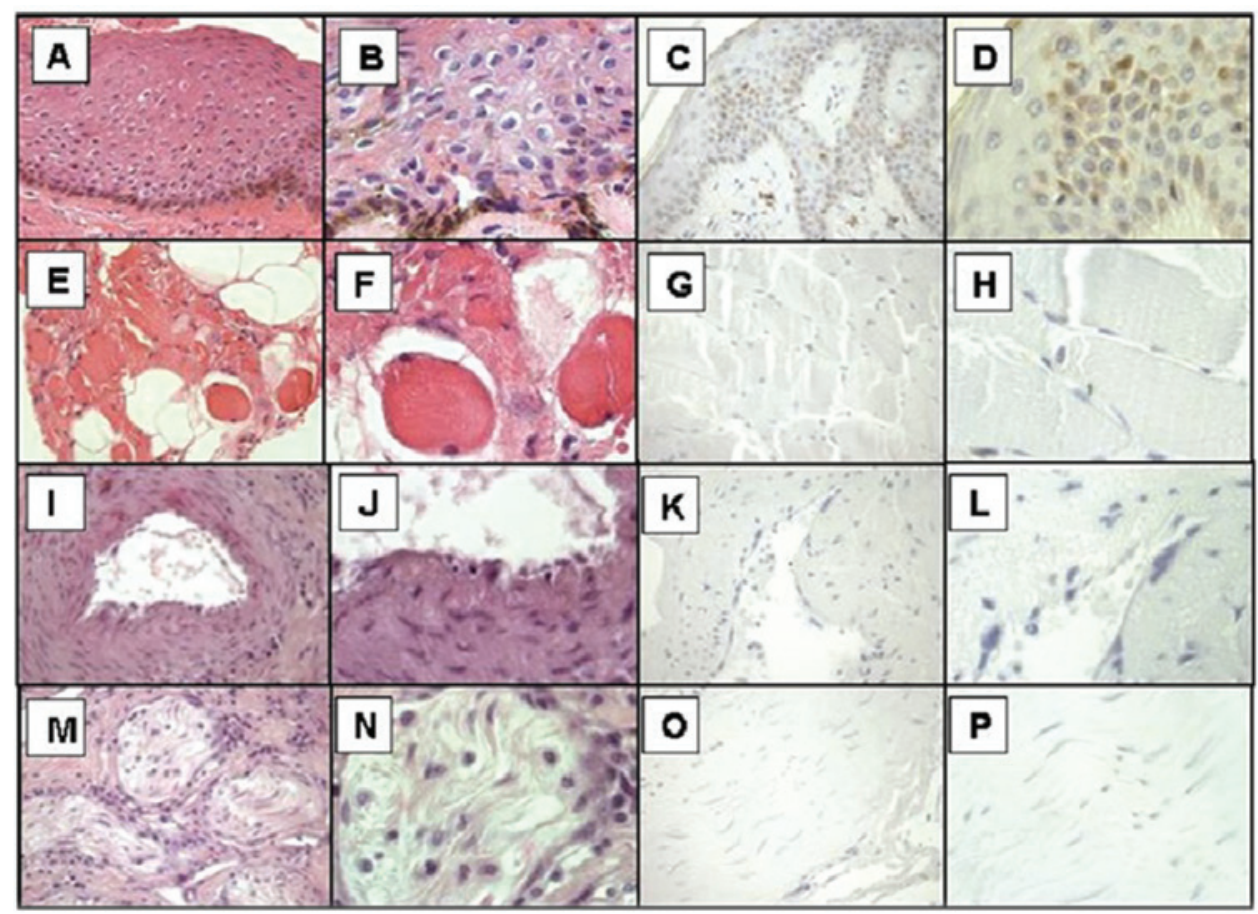

Figure 3. Representative tissues of varicose ulcers in non-diabetic patients. H\&E staining and TUNEL immunohistochemical staining. (A and B) H\&E and (C and D) TUNEL-stained epidermal border ulcers with keratinocyte hyperplasia and apoptotic changes; TUNEL positive due to repair and remodeling of the ulcer. (E and F) H\&E and (G and H) TUNEL-stained skeletal muscle with irregular atrophy without TUNEL-positive evidence. (I and J) H\&E and (K and L) TUNEL-stained blood vessels and endothelial cells also show similar changes; however, these are TUNEL negative. (M and N) H\&E and (O and P) TUNEL-stained peripheral nerves with partial atrophy, TUNEL negative. (Magnification, x40: A, C, E, G, I, K, M and O). (Magnification, x100: B, D, F, H, $\mathrm{J}, \mathrm{L}$ and $\mathrm{P})$. 


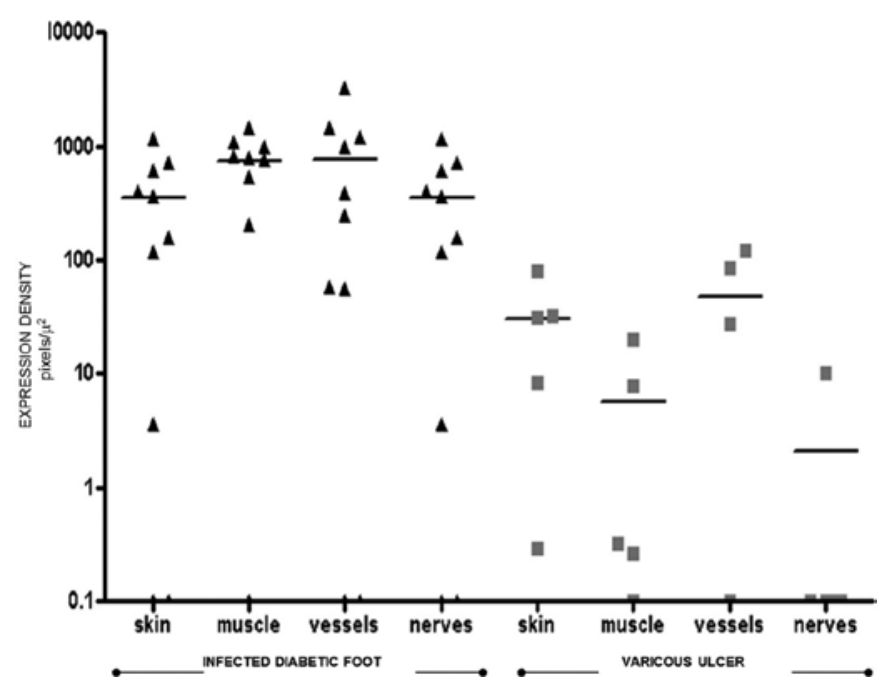

Figure 4. Quantification of TUNEL-positive cells in patients with infected diabetic foot or varicose ulcers. Computerized morphometric analysis of the TUNEL-positive cells in various tissues, of the surgical borders of the amputation were compared with samples from varicose ulcers. The magnitude of apoptosis was measured in skin, muscle, blood vessels and nerves.

considered free of necrosis or infection at the surgical borders of amputations required to treat infected diabetic foot. A total of 10 consecutive patients admitted to the hospital for amputation were studied. Of these, 2 patients denied that they had diabetes at the time of admission, and the rest had a 2 to 24-year history of DM2. This is a typical feature of the diabetic infected foot, since most cases occur in chronic poorly controlled patients (15), although this condition may also arise as the first manifestation of diabetes (16). The infectious process occurred 2-60 days prior to hospitalization, and the hospital length of stay was 7-150 days. The medical treatment required the use of broad-spectrum antibiotics, mainly thirdgeneration cephalosporins. A number of debriding surgeries were performed to eliminate the necrotic tissues prior to the decision to amputate. Although 6 patients were admitted with an APACHE II score $<5$, only 1 progressed without complications, whereas the rest had torpid evolution with symptoms of sepsis and 7 developed acute renal failure. This is explained by the fact that the Infectious Diseases Hospital receives severely ill patients referred by other hospitals. However, only 2 patients succumbed.

The 5 patients who required above-the-ankle amputation had an ankle-brachial pressure index $<0.45(0.34 \pm 0.10)$. This index provides a practical assessment of the severity of arterial occlusive disease, although it should be measured by an experienced physician and may provide incorrect information due to the distinctive rigid arterial walls of atherosclerosis $(17,18)$. The Doppler color and ultrasonography confirmed that all patients had varying degrees of occlusion of the femoral, popliteal, tibial and pedal arteries caused by atheromatous plaques and stenosis. The diabetic foot lesions displayed typical histological alterations of DM2 comprising necrotic and fibrotic tissues with inflammatory infiltration, granulation, angiogenesis and atherosclerotic plaques, and a marked decrease in the vascular lumen diameter and presence of apoptotic cells associated with wound healing.
The key observation from this study was that apoptotic cells were identified at the surgical borders of the amputations. This observation shows that damage is present in the tissues that the surgeon considered free of infection and necrosis. Apoptosis at this site is not explained by the woundhealing process since the samples were obtained during the surgery. Apoptotic cells were observed in the skin, skeletal muscle, blood vessels and peripheral nerves. As a control, the magnitude of the TUNEL-positive cells was compared with another condition and was 2-3 logarithms higher in the surgical samples from the DM2 patients compared to those from varicose ulcers obtained from the non-diabetic patients. The identification of apoptotic cells by TUNEL has the disadvantage of showing positive results in some necrotic cells, and pyroptosis, a form of programmed cell death associated with antimicrobial responses during inflammation, might explain the tissue damage (19-21).

Apoptosis plays a role in the tissue damage caused by chronic complications in DM2 (22-26). Apoptosis during acute complications of a chronic condition such as diabetic foot may be explained by at least 5 mechanisms: high glucose concentration (27-31), ketoacidosis, systemic inflammatory response (32), infection and hypoxia. The observation of apoptotic cells in the surgical borders may not have any clinical implication since it may be limited after the metabolic alterations and infection are controlled. However, if associated with hypoxia in the infected diabetic patient (IDP), the infected tissue could develop large zones of hypoxia, which may lead to gangrene. The infectious process progresses rapidly and may lead to necrotizing fasciitis, systemic inflammatory syndrome and multiorgan failure characteristic of sepsis. Necrosis is caused by the infection and hypoxia, and is characterized by the rapid loss of cellular homeostasis and the resultant edema, organelle disruption and early rupture of the cytoplasm membrane and efflux of the cellular content, which initiate an inflammatory response. Hypoxia could be aggravated by microemboli produced by atheromatous plaques.

A total of 4 patients had atheromatous plaques accompanied by macrophages and lymphocytes. Such plaques are considered vulnerable or high risk (33) due to their fragility and thrombogenic capacity, and they are characterized by a fibrous capsule, large number of macrophages and a nucleus rich in lipids, with an elevated content of proinflammatory factors (34). The infectious process in the IDP contributes to the development of apoptosis due to the action of lipopolysaccharide or proinflammatory cytokines such as tumor necrosis factor (35). Hyperglycemia increases the cytotoxic effect of LPS and ischemia (36). Similarly, plaques were able to stimulate apoptotic phagocytes, causing thrombosis, an inflammatory response and vascular injury (24). In the present study, regardless of the extent of tissue destruction, cells of varying cellular lineage were associated with apoptosis in the lesions obtained from IDPs. These cells included keratinocytes, fibroblasts, polymorphonuclear leukocytes, foamy macrophages and endothelial cells, apoptosis was also observed in muscular vessels.

The role of reperfusion in the induction of apoptosis is controversial. The experimental evidence suggests that the majority of cell death during ischemic events is caused by necrosis, whereas apoptosis appears only after reperfusion $(37,38)$ in cells that are 'compromised' and programmed 
to succumb during ischemia $(39,40)$. A number of studies have reported apoptosis in smooth muscle cells, atheromas and restenotic lesions in humans and mice $(41,42)$.

Apoptotic cells may appear during the process of regeneration in tissue and may involve endothelial, smooth muscular, macrophages, neutrophils and epidermal cells. Histopathological findings were expected since it is known that apoptosis plays a role in cicatrization. However, diabetic patients have defects in the process of cicatrization and in the resolution of injury due to cellular dysfunction and inadequate production of proteases, cytokines and growth factors. The activation of receptors for advanced glycation end products may decrease angiogenesis and increase apoptosis (43). By contrast, the inflammatory reaction in diabetic lesions with poor cicatrization appears to slow recovery by generating an intense proteolytic response, which is mediated by elastase and neutrophils, TNF and IL-1. This inflammatory reaction results from bacterial contamination and recurrent trauma in tissues; however, is not associated with pain $(15,18,26,44)$.

The apoptosis observed in these patients may be explained in numerous ways: preexisting metabolic acidosis, the initial ischemia, an inflammatory response to the infection induced by the trauma of surgery or anesthetics, which could increase the production of reactive oxygen species in the mitochondria $(45,46)$. In all patients in this study, apoptotic cells were identified and associated with vascular injury. It is likely that hypoxia was induced by apoptosis at the same time as unstable atheromatous plaques provoked acute obstruction of the arteries. The detection of apoptosis above the necrotic lesions and the vascular Doppler results support the decisions made during surgery to treat these IDPs.

\section{Acknowledgements}

The authors would like to thank Dr Rosario Mora-Campos, Dr Jorge Zendejas-Villanueva and QFB Lilia Flores-González for their technical assistance. This study was in part financed by CONACYT (no. 136 IP, Carolina Bekker Méndez) and FOFOI, IMSS (no. 2004/033 IP, César González Bonilla).

\section{References}

1. Wild S, Roglic G, Green A, Sicree R and King H: Global prevalence of diabetes: estimates for the year 2000 and projections for 2030. Diabetes Care 27: 1047-1053, 2004.

2. Giacco F and Brownlee M: Oxidative stress and diabetic complications. Circ Res 107: 1058-1070, 2010.

3. Brownlee M: Biochemistry and molecular cell biology of diabetic complications. Nature 414: 813-820, 2001.

4. Expert Committee on the Diagnosis and Classification of Diabetes Mellitus: Report of the expert committee on the diagnosis and classification of diabetes mellitus. Diabetes Care 25: S5-S20, 2002.

5. Mukhtar Q, Jack L Jr, Martin M, Murphy D and Rivera M: Evaluating progress toward healthy people 2010 national diabetes objectives. Prev Chronic Dis 3: A11, 2006.

6. Jones RN and Marshall WP: Does the proximity of an amputation, length of time between foot ulcer development and amputation, or glycemic control at the time of amputation affect the mortality rate of people with diabetes who undergo an amputation? Adv Skin Wound Care 21: 118-123, 2008.

7. Donley BG, Philbin T, Tomford JW and Sferra JJ: Foot and ankle infections after surgery. Clin Orthop 391: 162-170, 2001.

8. Schaper NC: Diabetic foot ulcer classification system for research purposes: a progress report on criteria for including patients in research studies. Diabetes Metab Res Rev 20: S90-S95, 2004.
9. Driver VR, Goodman RA, Fabbi M, French MA and Andersen CA: The impact of podiatric lead limb preservation team on disease outcomes and risk prediction in the diabetic lower extremity: a retrospective cohort study. J Am Podiatr Med Assoc 100: 235-241, 2010.

10. Lavery LA, Armstrong DG, Wunderlich RP, Mohler MJ, Wendel CS and Lipsky BA: Risk factors for foot infections in individuals with diabetes. Diabetes Care 29: 1288-1293, 2006.

11. Peters EJ, Armstrong DG and Lavery LA: Risk factors for recurrent diabetic foot ulcers: site matters. Diabetes Care 30: 2077-2079, 2007.

12. Resnick HE, Valsania P and Phillips CL: Diabetes mellitus and nontraumatic lower extremity amputation in black and white Americans: the National Health and Nutrition Examination Survey Epidemiologic Follow-up Study, 1971-1992. Arch Intern Med 159: 2470-2475, 1999.

13. Schofield CJ, Libby G, Brennan GM, MacAlpine RR, Morris AD and Leese GP: Mortality and hospitalization in patients after amputation: a comparison between patients with and without diabetes. Diabetes Care 29: 2252-2256, 2006.

14. Wagner FW Jr: The dysvascular foot: a system for diagnosis and treatment. Foot Ankle 2: 64-122, 1981.

15. Lepore G, Maglio ML, Cuni C, Dodesini AR, Nosari I, Minetti B and Trevisan R: Poor glucose control in the year before admission as a powerful predictor of amputation in hospitalized patients with diabetic foot ulceration. Diabetes Care 29: 1985, 2006.

16. New JP, McDowell D, Burns E and Young RJ: Problem of amputations in patients with newly diagnosed diabetes mellitus. Diabet Med 15: 760-764, 1998.

17. Ray SA, Srodon PD, Taylor RS and Dormandy JA: Reliability of ankle: brachial pressure index measurement by junior doctors. Br J Surg 81: 188-190, 1994.

18. Crawford F, Inkster M, Kleijnen J and Fahey T: Predicting foot ulcers in patients with diabetes: a systematic review and metaanalysis. QJM 100: 65-86, 2007.

19. Csizmadia E and Csizmadia V: Detection of apoptosis in tissue sections. Methods Mol Biol 539: 49-63, 2009.

20. Muppidi J, Porter M and Siegel RM: Measurement of apoptosis and and other forms of cell death. Curr Protoc Immunol (Chapt 3): 17, 2004.

21. Kroemer G, Galluzzi L, Vandenabeele P, et al: Classification of cell death: recommendations of the nomenclature committee on cell death 2009. Cell Death Differ 16: 3-11, 2009.

22. Sugiyama T, Kobayashi M, Kawamura H, Li Q and Puro DG: Enhancement of P2X(7)-induced pore formation and apoptosis: an early effect of diabetes on the retinal microvasculature. Invest Ophthalmol Vis Sci 45: 1026-1032, 2004.

23. Romeo G, Liu WH, Asnaghi V, Kern TS and Lorenzi M: Activation of nuclear factor- $\kappa \mathrm{B}$ induced by diabetes and high glucose regulates a proapoptotic program in retinal pericytes. Diabetes 51: 2241-2248, 2002.

24. Ramana KV, Friedrich B, Bhatnagar A and Srivastava SK: Aldose reductase mediates cytotoxic signals of hyperglycemia and TNF-alpha in human lens epithelial cells. FASEB J 17: 315-317, 2003 .

25. Hartz S, Menart B and Tschoepe D: Leukocyte apoptosis in whole blood involves platelet-dependent coaggregation. Cytometry A 52: 117-121, 2003.

26. Schmeichel AM, Schmelzer JD and Low PA: Oxidative injury and apoptosis of dorsal root ganglion neurons in chronic experimental diabetic neuropathy. Diabetes 52: 165-171, 2003.

27. Kang DH and Johnson RJ: Vascular endothelial growth factor: a new player in the pathogenesis of renal fibrosis. Curr Opin Nephrol Hypertens 12: 43-49, 2003.

28. Recchioni R, Marcheselli F, Moroni F and Pieri C: Apoptosis in human aortic endothelial cells induced by hyperglycemic condition involves mitochondrial depolarization and is prevented by N-acetyl-L-cysteine. Metabolism 51: 1384-1388, 2002.

29. Peiro C, Lafuente N, Matesanz N, Cercas E, Llergo JL, Vallejo S, Rodríguez-Mañas L and Sánchez-Ferrer CF: High glucose induces cell death of cultured human aortic smooth muscle cells through the formation of hydrogen peroxide. Br J Pharmacol 133: 967-974, 2001

30. Fiordaliso F,Leri A, Cesselli D, Limana F, Safai B, Nadal-Ginard B, Anversa P and Kajstura J: Hyperglycemia activates p53 and p53-regulated genes leading to myocyte cell death. Diabetes 50: 2363-2375, 2001

31. Cai L, Li W, Wang G, Guo L, Jiang Y and Kang YJ: Hyperglycemia-induced apoptosis in mouse myocardium: mitochondrial cytochrome $\mathrm{C}$-mediated caspase- 3 activation pathway. Diabetes 51: 1938-1948, 2002. 
32. Yasuhara S, Asai A, Sahani ND and Martyn JA: Mitochondria, endoplasmic reticulum, and alternative pathways of cell death in critical illness. Crit Care Med 35: S488-S495, 2007.

33. Esaki T, Hayashi T, Muto E, Kano H, Kumar TN, Asai Y, Sumi D and Iguchi A: Expression of inducible nitric oxide synthase and Fas/Fas ligand correlates with the incidence of apoptotic cell death in atheromatous plaques of human coronary arteries. Nitric Oxide 4: 561-571, 2000.

34. Fernandez-Ortiz A, Badimon JJ, Falk E, Fuster V, Meyer B, Mailhac A, Weng D, Shah PK and Badimon L: Characterization of the relative thrombogenicity of atherosclerotic plaque components: implications for consequences of plaque rupture. J Am Coll Cardiol 23: 1562-1569, 1994.

35. Liu R, Bal HS, Desta T, Behl Y and Graves DT: Tumor necrosis factor-alpha mediates diabetes-enhanced apoptosis of matrix-producing cells and impairs diabetic healing. Am J Pathol 168: 757-764, 2006.

36. Wang JY, Yang JM, Wang JY, Tao PL and Yang SN: Synergistic apoptosis induced by bacterial endotoxin lipopolysaccharide and high glucose in rat microglia. Neurosci Lett 304: 177-180, 2001.

37. Gottlieb RA, Gruol DL, Zhu JY and Engler RL: Preconditioning rabbit cardiomyocytes: role of $\mathrm{pH}$, vacuolar proton ATPase, and apoptosis. J Clin Invest 97: 2391-2398, 1996.

38. Webster KA, Discher DJ, Kaiser S, Hernandez O, Sato B and Bishopric NH: Hypoxia-activated apoptosis of cardiac myocytes requires reoxygenation or a $\mathrm{pH}$ shift and is independent of $\mathrm{p} 53$. J Clin Invest 104: 239-252, 1999.

39. Fliss H and Gattinger D: Apoptosis in ischemic and reperfused rat myocardium. Circ Res 79: 949-956, 1996.
40. Freude B, Masters TN, Robicsek F, Fokin A, Kostin S, Zimmermann R, Ullmann C, Lorenz-Meyer S and Schaper J: Apoptosis is initiated by myocardial ischemia and executed during reperfusion. J Mol Cell Cardiol 32: 197-208, 2000.

41. Bennett MR: Apoptosis of vascular smooth muscle cells in vascular remodelling and atherosclerotic plaque rupture. Cardiovasc Res 41: 361-368, 1999.

42. Isner JM, Kearney M, Bortman S and Passeri J: Apoptosis in human atherosclerosis and restenosis. Circulation 91: 2703-2711, 1995.

43. Shoji T, Koyama H, Morioka T, Tanaka S, Kizu A, Motoyama K Mori K, Fukumoto S, Shioi A, Shimogaito N, et al: Receptor for advanced glycation end products is involved in impaired angiogenic response in diabetes. Diabetes 55: 2245-2255, 2006.

44. Lobmann R, Schultz G and Lehnert H: Proteases and the diabetic foot syndrome: mechanisms and therapeutic implications. Diabetes Care 28: 461-471, 2005.

45. Yu T, Robotham JL and Yoon Y: Increased production of reactive oxygen species in hyperglycemic conditions requires dynamic change of mitochondrial morphology. Proc Natl Acad Sci USA 103: 2653-2658, 2006.

46. Sedlic F, Pravdic D, Ljubkovic M, Marinovic J, Stadnicka A and Bosnjak ZJ: Differences in production of reactive oxygen species and mitochondrial uncoupling as events in the preconditioning signaling cascade between desflurane and sevoflurane. Anesth Analg 109: 405-411, 2009. 